\title{
Health-related quality of life is not related to laparoscopic or robotic technique in radical cystectomy
}

\author{
*Kajetan Juszczak ${ }^{1, A, C-F}$, ${ }^{*}$ Agata Gastecka ${ }^{1, A-D}$, Jan Adamowicz, ${ }^{1, B-D}$, \\ Przemysław Adamczyk ${ }^{2, B-D}$, Marta Pokrywczyńska ${ }^{3, E, F}$, Tomasz Drewa ${ }^{1,2, E, F}$ \\ ${ }^{1}$ Department of General and Oncologic Urology, Collegium Medicum, Nicolaus Copernicus University, Bydgoszcz, Poland \\ 2 Department of General and Oncological Urology, Nicolaus Copernicus Hospital, Torú, Poland \\ ${ }^{3}$ Department of Regenerative Medicine Cell and Tissue Bank, Department of Urology, Collegium Medicum, Nicolaus Copernicus University, Bydgoszcz, Poland \\ A - research concept and design; $\mathrm{B}$ - collection and/or assembly of data; $\mathrm{C}$ - data analysis and interpretation; \\ $\mathrm{D}$ - writing the article; $\mathrm{E}$ - critical revision of the article; $\mathrm{F}$ - final approval of the article
}

\section{Address for correspondence \\ Agata Gastecka}

E-mail:agata.gastecka@gmail.com

\section{Funding sources}

The work was partly financed by the National Centre for Research and Development under the STRATEGMED I program "Prevention and Treatment of Lifestyle Diseases" project entitled "Innovative urine draining prothesis for patients with urinary bladder cancer treated with minimum invasive urinary bladder oncological surgeries" (STRATEGMED1/235368/8/NCBR/2014 (Smart AUCI)).

Conflict of interest

None declared

Acknowledgements

The authors would like to thank the National Centre for Research and Development for their support.

* Kajetan Juszczak and Agata Gastecka contributed equally to this work.

Received on March 30, 2020

Reviewed on April 15, 2020

Accepted on May 1,2020

Published online on July 28, 2020

Cite as

Juszczak K, Gastecka A, Adamowicz J, Adamczyk P, Pokrywczyńska M, Drewa T. Health-related quality of life is not related to laparoscopic or robotic technique in radical cystectomy. Adv Clin Exp Med. 2020;29(7):857-863. doi:10.17219/acem/121937

DOI

10.17219/acem/121937

\section{Copyright}

Copyright by Author(s)

This is an article distributed under the terms of the

Creative Commons Attribution 3.0 Unported (CC BY 3.0)

(https://creativecommons.org/licenses/by/3.0/)

\begin{abstract}
Background. Evaluating health-related quality of life (HR-QoL) is important in urological oncology.

Objectives. To evaluate the impact of minimally invasive radical cystectomy (RC) on the patients' QoL depending on the surgical strategy (laparoscopic compared to robotic).

Material and methods. The study involved 110 patients divided into 2 groups: group $1(n=65)$, qualified for robotic-assisted RC (RARC), and group 2 ( $n=45)$, qualified for laparoscopic RC (LRC). A prospective analysis of QoL was performed. In the study, we used sociodemographic data taken from the patients' medical records, and data from standardized questionnaires of QoL surveys entitled Functional Assessment of Cancer Therapy - General (FACT-G) and Functional Assessment of Cancer Therapy for Bladder Cancer (FACT-BI).

Results. In the patients who had undergone LRC, a statistically significant difference in HR-QoL was noted only for the subjective well-being (SWB) domain regarding family and social life: The patients had higher SWB values before surgery than after it. In the patients who had undergone RARC, statistically significant differences in HR-QoL were noted in 3 domains: 1) SWB - family and social life, 2) FACT-Bl assessment and 3) FACT-G assessment. Both before and after surgery, no statistically significant differences were found between the 2 groups for any of the HR-QoL domains.
\end{abstract}

Conclusions. The surgical technique of minimally invasive endoscopic RC used (laparoscopic or robotic) does not affect HR-QoL domains.

Key words: radical cystectomy, quality of life, urinary diversion, laparoscopic, robotic-assisted 


\section{Introduction}

In modern urological oncology, more and more emphasis is being placed nowadays on evaluating patients' healthrelated quality of life (HR-QoL). In general, the research largely focuses on patients with urinary bladder cancer who have been qualified for radical cystectomy (RC). ${ }^{1,2}$ The $\mathrm{RC}$ is the standard treatment for localized muscleinvasive bladder cancer. An integral part of RC is the creation of a urinary diversion after the cystectomy. Currently, 2 types of urinary diversion are commonly used: abdominal diversion (ureterocutaneostomy or ileal conduit) and urethral diversion (orthotopic bladder substitution). ${ }^{3}$ Urinary diversion rather than urinary bladder removal seems to have a significant impact on the patients' QoL, necessitating lifestyle changes and often causing stress to the patient.

The ultimate goal of HR-QoL assessment is to examine the impact of the type of treatment on the patient's health, taking into account their physical and emotional condition as well as social and functional domains. There are several instruments for HR-QoL assessment in patients with urinary bladder cancer. These tools are characterized by different development and validation phases and different contexts of use, depending on the depth of tumor infiltration, the applied therapeutic method or the urinary diversion technique after RC. Initial assessments of HR-QoL use basic tools designed to assess the overall QoL in patients with cancer: the European Organization for the Research and Treatment of Cancer Quality of Life Questionnaire (EORTC QLQ-C30) and the Functional Assessment of Cancer Therapy - General (FACT-G) questionnaire. ${ }^{4,5}$

The aim of the current study was to evaluate the impact of minimally invasive RC on the HR-QoL of patients depending on the surgical strategy used: laparoscopic compared to robotic.

\section{Material and methods}

\section{Study groups}

Out of 156 consecutive patients with muscle-invasive urinary bladder cancer, 110 were included in the study. The patients were divided into 2 groups according to the minimally invasive surgical technique used during RC. Group 1 consisted of 65 patients qualified for robotic-assisted RC (RARC); group 2 encompassed 45 patients qualified for laparoscopic RC (LRC). In each of the main groups, the patients were divided into subgroups depending on whether or not they had postoperative complications during their planned hospitalization. The division of the patients into these subgroups was based on the Clavien-Dindo classification.

The study was approved by the Ethics Committee of the Collegium Medicum, Nicolaus Copernicus University (Bydgoszcz, Poland; approval No. 60/2016 dated January 26, 2016, and the annex dated March 21, 2017).

\section{Inclusion and exclusion criteria}

The inclusion criteria for the study were: 1) urinary bladder cancer qualified for $\mathrm{RC}$; and 2) voluntary informed written consent to participate in the study. The exclusion criteria were: 1 ) RC due to neuromuscular urinary bladder dysfunction, prostate cancer or a primary tumor of an organ other than the urinary bladder; 2) changing the operating technique from LRC or RARC to open surgery, 3) senile dementia and/or the presence of a mental illness preventing an informed and voluntary decision regarding participation in the study; 4) confirmed homelessness; and 5) refusal to participate in the study for subjective reasons.

On the basis of the exclusion criteria, 46 patients out of 156 were not enrolled in the study, including 20 who underwent LRC and 26 who underwent RARC. Twelve patients from the LRC group did not agree to participate in the study, and the remaining 8 patients did not meet the inclusion criteria. In the non-included RARC group, 13 refused to participate and 13 were excluded.

\section{HR-QoL assessment}

A prospective analysis of QoL of the patients qualified for $\mathrm{RC}$ was performed. In order to find out the QoL of patients undergoing $\mathrm{RC}$, depending on the surgical technique used and long-term complications noted, the diagnostic survey method was used. In the study, we used sociodemographic data taken from medical records of patients and data from standardized questionnaires of QoL surveys: FACT-G, used to assess the physical condition, family and social life, emotional state and functioning in everyday life in patients with any type of cancer, and the Functional Assessment of Cancer Therapy - Bladder (FACT-Bl) to assess complaints and treatment related directly to urinary bladder cancer.

The FACT-Bl is a reliable, approved and flexible questionnaire used in many types and stages of urinary bladder cancer treatment developed by the Functional Assessment of Chronic Illness Therapy (FACIT) group. ${ }^{6}$ It differs from EORTC instruments in that it has been validated and designed to compare HR-QoL between patients with muscleinvasive bladder cancer (MIBC) and those with non-muscle-invasive bladder cancer (NMIBC). The form consists of 27 questions from the general version of the FACT-G questionnaire, divided into 4 domains - physical, social/family, emotional, and functional - accompanied by 12 questions specific to urinary bladder cancer. These questions relate to urine function, bowel function, sexual function, body image, appetite and stoma care, and are rated on a scale of 0 to 4 . The bladder-specific answers are added together and added to the FACT-G result. A high number of points indicates better HR-QoL. All the questions in the survey relate to the patient's HR-QoL over the previous week.

On the basis of the FACT-G form, a questionnaire entitled Functional Assessment of Cancer Therapy - Bladder Cystectomy (FACT-Bl) formerly known as the Venderbilt 
Cystectomy Index (FACT-VCI), was also developed. It is a validated tool designed for HR-QoL measurement in patients undergoing radical cystectomy. The questionnaire reserved for patients with urinary bladder cancer focuses mainly on clinical symptoms related to urinary bladder, gastro-intestinal and sexual dysfunction after radical cystectomy. $^{6-9}$

The FACIT group approved the use of the FACT-G and FACT-Bl questionnaires in Polish. The prospective measurements were taken twice: before surgery and within a period of $\leq 12$ months after surgery. Before surgery, the patients completed the questionnaires given to them in the hospital by a researcher. The $2^{\text {nd }}$ measurement was done using the postal survey method or with the Computer-Assisted Telephone Interviewing (CATI) method.

\section{Statistical analysis}

The results are expressed as means \pm standard deviation (SD). To check the significance of differences in the level of the QoL before and after the procedures, a parametric Student's t-test for dependent samples (when the condition of difference distribution normality was met) and a non-parametric Wilcoxon pair order test (when there was non-normal distribution) were used. Statistical significance was set at $\mathrm{p} \leq 0.05$ for all the tests. The statistical calculations were performed using STATISTICA v. 10 PL software (StatSoft Polska, Kraków, Poland).

\section{Results}

\section{Group characteristics}

The results showed that the baseline health status of the patients in group 1 (RARC) was worse than in the patients in group 2 (LRC). In group 2, 53\% of the patients were at the pTa-pT 2 stage of cancer progression according to the TNM scale; group 1 included a 4\% lower rate of patients at this stage of the disease (49\%). A higher percentage of patients at the advanced pT3-pT4 stage of cancer was observed in group 1 (51\%) than in group 2 (47\%). The patients in group 1 were characterized by a more frequent occurrence of comorbidities; the differences between the 2 groups were $6 \%$ for cardiovascular diseases and $11 \%$ for diabetes. There were no statistically significant differences between the 2 groups in terms of body mass index (BMI) or American Society of Anesthesiologists (ASA) physical status scores.

Group 1 (RARC) included 16 women and 49 men with a mean age of 70 years (range: $52-87$ years). The subgroup of RARC patients who had no postoperative complications during their planned hospitalization was made up of 17 patients, including 13 men and 4 women. The subgroup of RARC patients with postoperative complications during their planned hospitalization comprised 48 cases, including 36 men and 12 women. A total of 48 group 1 patients survived the one-year follow-up period: 20 patients with ureterocutaneostomy (42\%) and 28 patients with ileal conduits (58\%).

Group 2 (LRC) included 6 women and 39 men with a mean age of 66 years (range: $43-86$ years). The subgroup of LRC patients who had no postoperative complications during their planned hospitalization consisted of 14 people (13 men and 1 woman). The subgroup of LCR patients who had complications during their planned hospitalization consisted of 31 patients, including 26 men and 5 women. A total of 27 group 2 patients survived the one-year followup period: 16 patients with ureterocutaneostomy (59\%) and 11 patients with ileal conduits (41\%).

\section{Hospitalization time}

Our results revealed no significant relationship between the surgical technique used and hospitalization time. In group 1, the hospitalization time was shorter by an average of 2 days compared to group 2. The duration of hospitalization in the subgroup of patients in whom no postoperative complications were noted was the same for both analyzed groups. The greatest difference in the length of hospital stay following RC surgery was observed in the subgroups of patients with postoperative complications; the time was shorter for the RARC group (12 days) compared to the LRC group (16 days). The results obtained in this regard are consistent with the results regarding the scale of complications. Patients from the RARC group had a lower rate of Grade III-V complications (Clavien-Dindo Scale) compared to the LRC technique, resulting in a shorter hospitalization.

\section{Urinary diversion}

In the group of patients operated on using the LRC technique, ureterocutaneostomy (71\%) was most often chosen as a method of urinary diversion. In the patients operated on with the RARC technique, the urinary diversion methods used were equally divided (51\% for ureterocutaneostomy compared with $49 \%$ for ileal conduit). The study showed a statistically significant difference in the method of reconstruction of the urinary tract between the LRC and RARC groups ( $p=0.0329)$. A higher percentage of urinary diversion procedures using an ileal fragment (49\%) was noted among patients after RARC. A statistically significant relationship between the surgical technique and the urinary diversion procedure was also found in the subgroup of patients with postoperative complications $(\mathrm{p}=0.0281)$.

\section{HR-QoL assessment}

The 48 RARC patients and 27 LRC patients who survived the one-year follow-up period were included in the postoperative HR-QoL assessment. In both groups, males, 
people living in urban areas and respondents receiving retirement benefits constituted larger shares. The average age in the LRC group was 64 years (range: $54-75$ years); the average age in the RARC group was 68 years (range: $52-87$ years).

The results regarding the clinical data of the patients included in the QoL assessment after RC surgery did not show any significant differences between the 2 groups. In the LRC group, $59 \%$ of the patients underwent ureterocutaneostomy $(n=16)$ and $41 \%$ ileal conduits $(n=11)$. In the RARC group, $42 \%$ of the patients underwent ureterocutaneostomy $(n=20)$ and $58 \%$ ileal conduits $(n=28)$. Among the respondents who assessed their HR-QoL after
RC surgery, $40 \%$ of those in the RARC group and 30\% in the LRC group had pT3-pT4 level cancer. Patients from the RARC group who survived the one-year follow-up were characterized by higher rates of comorbidities than the patients who had undergone LRC.

In the patients who had undergone LRC, a statistically significant difference in HR-QoL occurred only for the subjective well-being (SWB) domain regarding family and social life $(\mathrm{p}=0.0487)$. All of these patients had higher SWB values before surgery than after surgery. For other QoL domains, no statistically significant differences were found between the results before and after surgery (p > 0.05) (Table 1).

Table 1. Comparison of HR-QoL domains in patients before and after minimally invasive RC

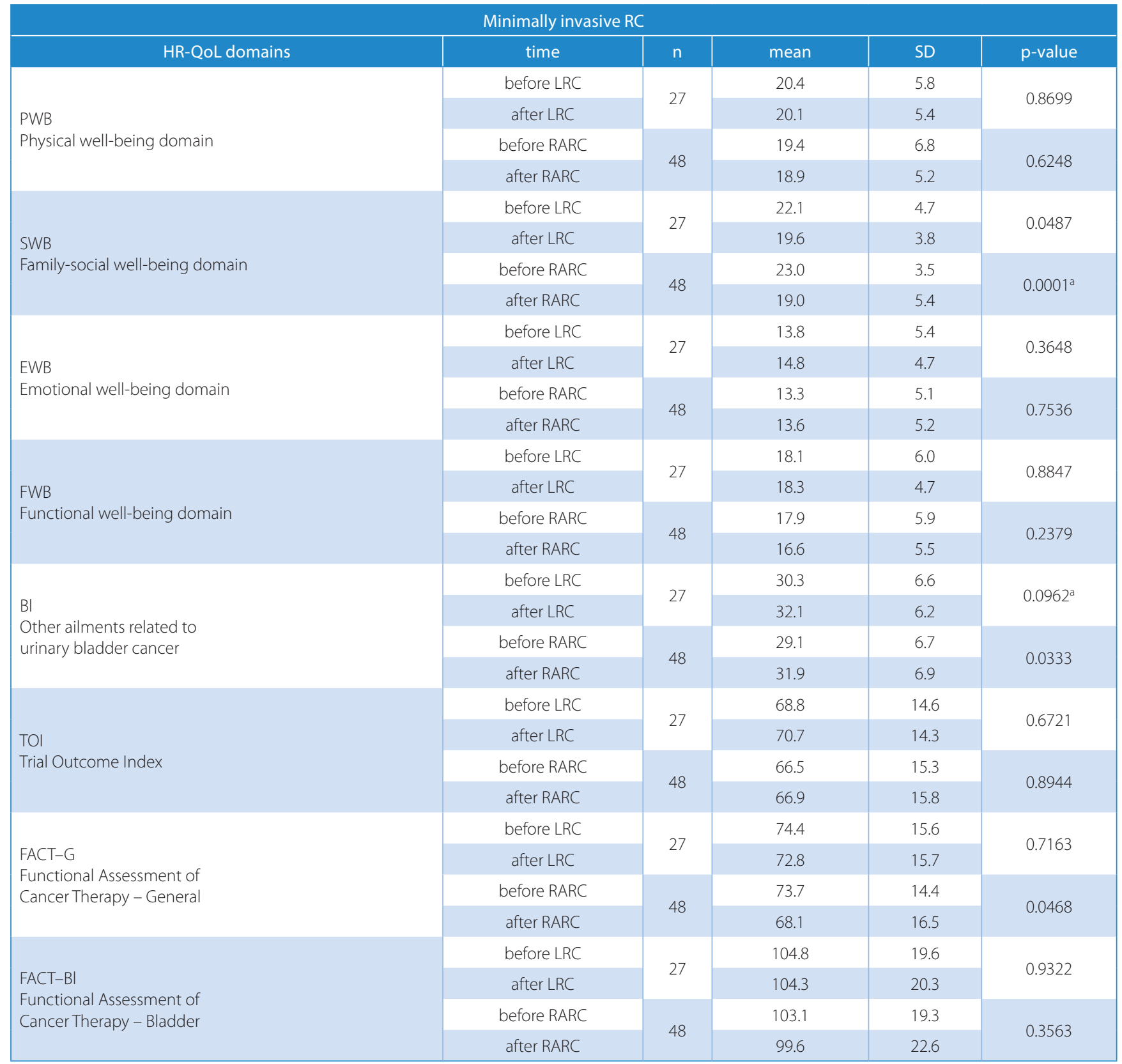

aWilcoxon pair order test result; HR-QoL - health-related quality of life; RC - radical cystectomy; LRC - laparoscopic radical cystectomy; RARC - roboticassisted radical cystectomy; SD - standard deviation; $n$ - number of patients. 
In the patients who had undergone RARC, statistically significant differences in the HR-QoL were found for the following domains: 1) the SWB domain concerning family and social life ( $\mathrm{p}<0.0001)$, where the patients had higher SWB values before surgery than after surgery; 2) FACT-Bl ( $p=0.0333)$, where the patients had higher values after the procedure than before the procedure; and 3) FACT-G ( $p=0.0468)$, where the patients had higher values before surgery than after it. For the remaining QoL domains, no statistically significant differences were found between the results before and after surgery $(p>0.05)$ (Table 1).
Both before and after surgery, no statistically significant differences were found between the RARC patients and the LRC patients for any of the HR-QoL domains ( $p>0.05$ ). The HR-QoL of the patients in both groups was assessed at a similar level before RC surgery (Table 2).

\section{Discussion}

Health-related QoL assessment plays a significant role in the treatment of oncological diseases. In addition to ascertaining whether and how patients benefit from

Table 2. Comparison of HR-QoL domains in patients before and after minimally invasive radical cystectomy (LRC compared with RARC)

\begin{tabular}{|c|c|c|c|c|c|}
\hline \multicolumn{6}{|c|}{ Minimally invasive radical cystectomy } \\
\hline HR-QoL domains & time & $\mathrm{n}$ & mean & $\mathrm{SD}$ & $p$-value \\
\hline \multirow{4}{*}{$\begin{array}{l}\text { PWB } \\
\text { Physical well-being domain }\end{array}$} & before LRC & 27 & 20.4 & 5.8 & \multirow{2}{*}{$0.6662^{(a)}$} \\
\hline & before RARC & 48 & 19.4 & 6.8 & \\
\hline & after LRC & 27 & 20.1 & 5.4 & \multirow{4}{*}{0.3516} \\
\hline & after RARC & 48 & 18.9 & 5.2 & \\
\hline \multirow{4}{*}{$\begin{array}{l}\text { SWB } \\
\text { Family-social well-being domain }\end{array}$} & before LRC & 27 & 22.1 & 4.7 & \\
\hline & before RARC & 48 & 23.0 & 3.5 & \\
\hline & after LRC & 27 & 19.6 & 3.8 & \multirow{4}{*}{0.6786} \\
\hline & after RARC & 48 & 19.0 & 5.4 & \\
\hline \multirow{4}{*}{$\begin{array}{l}\text { EWB } \\
\text { Emotional well-being domain }\end{array}$} & before LRC & 27 & 13.8 & 5.4 & \\
\hline & before RARC & 48 & 13.3 & 5.1 & \\
\hline & after LRC & 27 & 14.8 & 4.7 & \multirow{4}{*}{0.8926} \\
\hline & after RARC & 48 & 13.6 & 5.2 & \\
\hline \multirow{4}{*}{$\begin{array}{l}\text { FWB } \\
\text { Functional well-being domain }\end{array}$} & before LRC & 27 & 18.1 & 6.0 & \\
\hline & before RARC & 48 & 17.9 & 5.9 & \\
\hline & after LRC & 27 & 18.3 & 4.7 & \multirow{4}{*}{$0.4759^{(a)}$} \\
\hline & after RARC & 48 & 16.6 & 5.5 & \\
\hline \multirow{4}{*}{$\begin{array}{l}\mathrm{Bl} \\
\text { Other ailments related to } \\
\text { urinary bladder cancer }\end{array}$} & before LRC & 27 & 30.3 & 6.6 & \\
\hline & before RARC & 48 & 29.1 & 6.7 & \\
\hline & after LRC & 27 & 32.1 & 6.2 & \multirow{4}{*}{0.5276} \\
\hline & after RARC & 48 & 31.9 & 6.9 & \\
\hline \multirow{4}{*}{$\begin{array}{l}\text { TOI } \\
\text { Trial Outcome Index }\end{array}$} & before LRC & 27 & 68.8 & 14.6 & \\
\hline & before RARC & 48 & 66.5 & 15.3 & \\
\hline & after LRC & 27 & 70.7 & 14.3 & \multirow{4}{*}{0.8484} \\
\hline & after RARC & 48 & 66.9 & 15.8 & \\
\hline \multirow{4}{*}{$\begin{array}{l}\text { FACT-G } \\
\text { Functional Assessment of } \\
\text { Cancer Therapy - General }\end{array}$} & before LRC & 27 & 74.4 & 15.6 & \\
\hline & before RARC & 48 & 73.7 & 14.4 & \\
\hline & after LRC & 27 & 72.8 & 15.7 & \multirow{4}{*}{0.7192} \\
\hline & after RARC & 48 & 68.1 & 16.5 & \\
\hline \multirow{4}{*}{$\begin{array}{l}\text { FACT-BI } \\
\text { Functional Assessment of } \\
\text { Cancer Therapy - Bladder }\end{array}$} & before LRC & 27 & 104.8 & 19.6 & \\
\hline & before RARC & 48 & 103.1 & 19.3 & \\
\hline & after LRC & 27 & 104.3 & 20.3 & \multirow{2}{*}{0.3760} \\
\hline & after RARC & 48 & 99.6 & 22.6 & \\
\hline
\end{tabular}

aMann-Whitney U test result; HR-QoL - health-related quality of life; RC - radical cystectomy; LRC - laparoscopic radical cystectomy; RARC - roboticassisted radical cystectomy; SD - standard deviation; $\mathrm{n}$ - number of patients. 
the treatment, assessing QoL facilitates the planning and implementation of long-term care. A relationship between the clinical stage of oncological disease, the type of treatment and the patients' QoL has been observed. Patients with chronic disease adjust to the current situation faster by lowering their expectations. ${ }^{10}$

Ureterocutaneostomy is the simplest form of incontinent cutaneous diversion. Operating times, complication rates, the duration of stays in intensive care, and the length of hospital stays are lower in patients treated with ureterocutaneostomy as compared to ileal conduits. ${ }^{11}$ Therefore, in older or compromised patients who need a supravesical diversion, ureterocutaneostomy is the preferred procedure. The basic assumptions gained from previous studies are that patients with continent cutaneous or orthotopic diversions may have improved QoL compared to those with incontinent ileal conduit urinary diversions. ${ }^{11}$ In a study by Gilbert et al., the QoL of patients who had undergone ileal conduit and ureterocutaneostomy procedures was assessed using the Bladder Cancer Index (BCI), which showed equal urinary bother and function scores for both groups of patients. ${ }^{12}$ Others have demonstrated that in carefully selected elderly patients, all forms of wet and dry urinary diversions, including orthotopic bladder substitutions, are possible. ${ }^{13}$

Quality of life is a dynamic, multidimensional construct that changes over time and can be affected by the experience of both the patients and the healthcare providers. The HR-QoL assesses the impact of illness on the subject's life and is considered an important component of patients' attitudes to their cancer. The range of parameters assessed with an HR-QoL questionnaire includes physical function, symptoms, global judgment of health, psychological and social well-being, cognitive functioning, roles and activities, personal attitudes, and satisfaction with care. ${ }^{14-16}$ Patients' acceptance of a stoma and rehabilitation in cases of ileal conduit diversion varies widely, but generally it can be said that urinary diversion is not an operation that renders the subject an invalid; psychosocial reintegration is possible, and adequate preoperative information, particularly on sexual status, is very important. ${ }^{17}$ Regarding postoperative status, patients with an ileal conduit usually have the poorest self-image, defined by a decrease in sexual desire and all forms of sexual and nonsexual physical contact. ${ }^{17}$ The advantages of various forms of continent urinary diversion are the presumed improvements in QoL compared with conduit diversion. However, continent urinary diversion may have some disadvantages as well. From a technical point of view, continent forms of diversion are more challenging and time-consuming, and patients leave the hospital with indwelling catheters. Once the catheters are removed, the patients must undergo a period of education in the techniques required to properly care for the reservoir or neobladder. Patients are also at a higher risk for diarrhea and vitamin B12 malabsorption. However, it is generally believed that the QoL advantages of continent urinary diversions outweigh the potential disadvantages..$^{18,19}$

Our results revealed that the patients who had undergone LRC were distinguished by slightly higher value indices for individual domains making up the overall QoL assessment than those who had undergone RARC. In the respondents who had undergone RARC, attention should be paid to the higher number of patients whose urine was drained using an ileal conduit, the higher number of patients with cancer at the pT3-pT4 level and the higher percentage of patients who were re-hospitalized due to complications than in the LRC group. The respondents who underwent RARC were on average 4 years older than the patients who had LRC. Despite the worse clinical data of the patients in the RARC group, no significant differences were found between the 2 study groups in the subjective assessments of QoL either before or after surgery. Moreover, when interpreting the results for the subjective assessment of HR-QoL, attention should be paid to the guidelines on the minimum and maximum score possible for each domain. The highest achievable result, indicating the best perceived QoL, for the HR-QoL domain for cancer (FACTG), is 108 points, as opposed to 156 points for the domain testing HR-QoL for urinary bladder cancer (FACT-Bl).

For patients from the LRC group before surgery, the FACT-G QoL was rated 31\% lower than the maximum value, i.e., 33.6 points on average. After surgery, the indicator fluctuated around 33\%, i.e., on average 35.2 points lower than the maximum possible. On FACT-Bl, the points scored were $33 \%$ (51 points) lower than the maximum before surgery and 33\% (52 points) lower after surgery. The patients in the RARC group scored 32\% (34 points) below the possible maximum on FACT-G before surgery and $37 \%$ (40 points) below the maximum value after surgery. On FACT-Bl, the RARC patients scored 34\% (53 points) lower than the possible maximum before the procedure and 36\% (56 points) lower after the procedure.

Our observations regarding the assessment of the LRC patients' QoL before and after surgery showed a significant decrease in the value of the family and social life domain $(p=0.0487)$. Lower values after surgery were also noted for the domain dealing with the patient's psychological wellbeing. Similarly, the assessment of the RARC patients' QoL before surgery compared to the one-year follow-up showed a significant difference for the family and social life domain $(p=0.0001)$. In addition, deterioration in the physical condition and functioning in everyday life of patients was observed. A significant difference was noted in the RARC patients' FACT-Bl results, which were better after surgery $(\mathrm{p}=0.0333)$, and in FACT-G results, which were worse after the observation period ( $p=0.0468$ ). Analysis of the FACT$\mathrm{G}$ and FACT-Bl responses shows that the patients in both study groups rated their HR-QoL not much worse after the $\mathrm{RC}$ procedure than before it.

There are few reports in the literature dealing with patient's QoL after RC surgery in relation to the surgical 
technique used. Most authors compare assessments of QoL for continent and incontinent urinary diversions. Bochner et al. indicated that there are no significant differences in the QoL of patients who had undergone open $\mathrm{RC}$ and those who had had RARC, taking into account follow-up periods of 3 and 6 months. ${ }^{20}$ Messer et al. reported that HR-QoL returned to the baseline 3 months after RC surgery, with no difference between open RC and RARC. ${ }^{21}$

The one-year follow-up period adopted in our study may have contributed to obtaining slightly better indicators for the QoL domains those mostly regarding emotional states. Assessing QoL a full year after RC surgery could have resulted in patients accepting their cancer and adapting to their changed body and life conditions. There were decreases in the value of domains concerning physical condition and social life in both groups of patients (LRC and RARC).

The results obtained on FACT-Bl are also noteworthy: The values noted for patients from both the LRC and RARC groups were higher at the $2^{\text {nd }}$ measurement, although one might expect that the necessity of living with a stoma, sexual dysfunction or scarring would significantly reduce the score. Positive adaptation to life with a stoma, and thus a better perception of QoL, largely depends on the patient's proficiency in urostomy care. ${ }^{22-24}$ According to observations reported by Kristensen et al., in order to ensure a sense of self-sufficiency, urostomy care requires manual skill and adaptive in the emotional sphere. ${ }^{25}$ Providing the patient with relevant information about the stoma and education in its use and care are extremely important elements of the process of improving the patients' self-sufficiency after surgery and their perception of their QoL. ${ }^{26,27}$ Performing RC procedures using minimally invasive techniques does not negatively affect the patients' self-image.

\section{Conclusions}

The results of our study indicate that the choice of minimally invasive endoscopic RC used (LRC compared to RARC) does not affect patients' HR-QoL.

\section{ORCID iDs}

Kajetan Juszczak (1) https://orcid.org/0000-0003-0354-0822 Agata Gastecka [1] https://orcid.org/0000-0002-3214-2287 Jan Adamowicz (1) https://orcid.org/0000-0003-2479-7133 Przemysław Adamczyk (1) https://orcid.org/0000-0002-7217-841X Marta Pokrywczyńska @ i https://orcid.org/0000-0003-3259-087X Tomasz Drewa (10) https://orcid.org/0000-0001-5347-4136

\section{References}

1. Moncrief TJ, Balaji P, Lindgren BB, Weight CJ, Konety BR. Comparative evaluation of bladder-specific Health-Related Quality of Life (HRQOL) instruments for bladder cancer. Urology. 2017;108:76-81.

2. Sosnowski R, Kulpa M, Ziętalewicz $U$, et al. Basic issues concerning health-related quality of life. Cent European JUrol. 2017;70(2):206-211.

3. Stein JP, Lieskovsky G, Cote R, et al. Radical cystectomy in the treatment of invasive bladder cancer: Long-term results in 1,054 patients. J Clin Oncol. 2001;19(3):666-675.
4. Webster K, Cella D, Yost K. The Functional Assessment of Chronic IIIness Therapy (FACIT) measurement system: Properties, applications, and interpretation. Health Qual Life Outcomes. 2003;16(1):79.

5. Danna BJ, Metcalfe MJ, Wood EL, Shah JB. Assessing symptom burden in bladder cancer: An overview of bladder cancer-specific HealthRelated Quality of Life instruments. Bladder Cancer. 2016;2(3):329-340.

6. Degboe A, Ivanescu C, Rohay JM, Turner RR, Cella D. Validity and performance of the Functional Assessment of Cancer Therapy - Bladder (FACT-BI) among advanced urothelial cancer patients. Support Care Cancer. 2019;27(11):4189-4198.

7. Mason SJ, Downing A, Wright $P$, et al. Health-related quality of life after treatment for bladder cancer in England. Br J Cancer. 2018;118(11): 1518-1528.

8. Anderson CB, Feurer ID, Large MC, et al. Psychometric characteristics of a condition-specific, health-related quality-of-life survey: The FACT-Vanderbilt Cystectomy Index. Urology. 2012;80(1):77-83.

9. Cookson MS, Dutta SC, Chang SS, Clark T, Smith JA Jr, Wells N. Healthrelated quality of life in patients treated with radical cystectomy and urinary diversion for urothelial carcinoma of the bladder: Development and validation of a new disease specific questionnaire. $J$ Urol. 2003;170(5):1926-1930.

10. Radecka B. Health-related quality of life: The role and manners of assessment in cancer patients. Curr Gynecol Oncol. 2015;13(3):172-179.

11. Parkinson JP, Konety BR. Health-related quality of life assessments for patients with bladder cancer. J Urol. 2004;172(6 Pt 1):2130-2136.

12. Gilbert SM, Wood DP, Dunn RL, et al. Measuring health-related quality of life outcomes in bladder cancer patients using the Bladder Cancer Index (BCI). Cancer. 2007;109(9):1756-1762.

13. Shih C, Porter MP. Health-related quality of life after cystectomy and urinary diversion for bladder cancer. Adv Urol. 2011;2011:715892.

14. Saber A. Urinary diversion: Historical aspect and patient's satisfaction. Urol Nephrol Open Access J. 2014;1(3):86-93.

15. Somani BK, MacLennan SJ, N'Dow J. Quality of life with urinary diversion. Eur Urol Supp. 2010;9(10):763-771.

16. Varricchio CG, Ferrans CE. Quality of life assessments in clinical practice. Semin Oncol Nurs. 2010;26(1):12-17.

17. Asgari MA, Safarinejad MR, Shakhssalim N, Soleimani M, Shahabi A, Amini E. Quality of life after radical cystectomy for bladder cancer in men with an ileal conduit or continent urinary diversion: A comparative study. Urol Ann. 2013;5(3):190-196.

18. Saika T, Arata R, Tsushima T, et al; Okayama Urological Research Group. Health-related quality of life after radical cystectomy for bladder cancer in elderly patients with an ileal conduit, ureterocutaneostomy, or orthotopic urinary reservoir: A comparative questionnaire survey. Acta Med Okayama. 2007;61(4):199-203.

19. Porter MP, Penson DF. Health-related quality of life after radical cystectomy and urinary diversion for bladder cancer: A systematic review and critical analysis of the literature. J Urol. 2005;173(4):1318-1322.

20. Bochner BH, Dalbagni G, Sjoberg DD, et al. Comparing open radical cystectomy and robot-assisted laparoscopic radical cystectomy: A randomized clinical trial. Eur Urol. 2015;67(6):1042-1050.

21. Messer JC, Punnen S, Fitzgerald J, Svatek R, Parekh DJ. Health-related quality of life from a prospective randomised clinical trial of robot-assisted laparoscopic vs open radical cystectomy. BJU Int. 2014;114(6):896-902.

22. Jensen BT, Kiesbye B, Soendergaard I, Jensen JB, Ammitzboell Kristensen $\mathrm{S}$. Efficacy of preoperative uro-stoma education on self-efficacy after radical cystectomy: Secondary outcome of a prospective randomized controlled trial. Eur J Oncol Nurs. 2017;28:41-46.

23. Danielsen AK, Rosenberg J. Health-related quality of life may increase when patients with a stoma attend patient education: A case-control study. PLoS One. 2014;9(3):e90354.

24. O'Connor G. Teaching stoma-management skills: The importance of self-care. Br J Nurs. 2005;14(6):320-324.

25. Kristensen SA, Laustsen S, Kiesbye B, Jensen BT. The Urostomy Education Scale: A reliable and valid tool to evaluate urostomy self-care skills among cystectomy patients. J Wound Ostomy Continence Nurs. 2013;40(6):611-617.

26. Kozell K, Frecea M, Thomas JT. Preoperative ostomy education and stoma site marking. J Wound Ostomy Continence Nurs. 2014;41(3): 206-207.

27. Fujimura T. Current status and future perspective of robot-assisted radical cystectomy for invasive bladder cancer. Int J Urol. 2019;26(11): 1033-1042. 